\title{
New variable stars in the globular cluster NGC 288
}

\author{
J. Kaluzny ${ }^{1}$, W. Krzemiński ${ }^{2}$, and M. Nalezyty ${ }^{1}$ \\ 1 Warsaw University Observatory, Al. Ujazdowskie 4, 00-478 Warsaw, Poland \\ e-mail: (jka,nalezyty)@sirius.astrouw.edu.pl \\ ${ }^{2}$ Carnegie Observatories, Las Campanas Observatory, La Serena, Cailla 601, Chile \\ e-mail: wojtek@roses.ctio.noao.edu
}

Received January 20; accepted January 22, 1997

\begin{abstract}
We report discovery of 3 new faint variables in the globular cluster NGC 288: 2 SX Phe stars and 1 contact binary. Both SX Phe variables are blue stragglers. The contact binary is located below cluster turnoff, slightly to the red of the main sequence. New photometry of 6 previously known variables is also presented. We note that 26 out of a total 43 SX Phe stars identified recently in $\omega$ Cen and NGC 288 exhibit $V$-band light curves with full amplitudes smaller than 0.10 mag. The sample of known SX Phe stars is likely to be significantly incomplete in regard to the low amplitude variables ${ }^{1}$.
\end{abstract}

Key words: globular clusters: individual: NGC 288 - stars: variables: other — blue stragglers stars: horizontal-branch - binaries: close

\section{Introduction}

This contribution continues a series of papers presenting results of an ongoing photometric survey for faint variables in southern globular clusters. Our main objective is the identification of detached eclipsing binaries suitable for accurate determination of turnoff masses and distances to the clusters (e.g. Paczyński 1996). As expected, the survey provides a rich harvest of contact binaries and SX Phe stars. Recently we reported discovery of a few dozen variables in $\omega$ Cen (Kaluzny et al. 1996, 1997), NGC 6397 (Kaluzny 1997) and M4 (Kaluzny, et al. 1997).

NGC $288(l=149 \mathrm{deg}, b=-89.4 \mathrm{deg})$ is one of the closest globular clusters. Low central concentration, very small reddening and a high galactic latitude make it a very

\footnotetext{
Send offprint requests to: J. Kaluzny

* Based on observations collected at Las Campanas Observatory of the Carnegie Institution of Washington.

1 Tables 2-11 are available only in electronic form at the CDS via anonymous ftp to cdsarc.u-strasbg.fr (130.79.128.5) or via http: //cdsweb.u-strasbg.fr/Abstract.html
}

attractive object for all sort of studies. The cluster harbors a large number of blue stragglers (Bolte 1992). Recently Kaluzny (1996; Paper I) reported on a pilot survey which resulted in the discovery of $4 \mathrm{SX}$ Phe stars and $1 \mathrm{RR}$ Lyr variable in the cluster. That data set consisted of $81 \mathrm{~V}$ band images collected with the CTIO 0.9-m telescope over a 13 day period in July 1995. Below we present results of a more extended survey of NGC 288 which was conducted at Las Campanas Observatory.

\section{Observations and data reduction}

The field centered approximately on the center of NGC 288 was monitored at Las Campanas Observatory with the 1-m Swope telescope and with the 2.5-m duPont telescope. The observational data were collected during several runs conducted between December 1990 and September 1992. The data were taken on 25 nights and on 7 nights the cluster was monitored continuously for at least 4.5 hours. Three different cameras were used: a) $1024^{2}$ Tektronix CCD with scale 0.61 " / pix - TEK2 camera, 1-m telescope; b) $2048^{2}$ Ford Aerospace CCD with scale 0.435 " / pix - FORD camera, 1-m telescope; c) $2048^{2}$ Tektronix CCD with scale $0.23 " /$ pix - TEK3 camera, 2.5-m telescope. Most of data were taken with the $V$ filter, with only occasional exposures taken in the $B$ and $I$ bands. We obtained a total of 462 useful images in the $V$-band. The exposure time ranged from 7 minutes to 10 minutes. For the prevailing number of images the seeing was better than 1.5 arcsec. A detailed log of all observations was submitted to the editors of A\&A (see Appendix A).

The preliminary processing of the raw data was made with $\mathrm{IRAF}^{2}$. Stellar photometry was extracted using DAOPHOT/ALLSTAR (Stetson 1987) and DoPHOT (Schechter et al. 1993) programs. We followed procedures

2 IRAF is distributed by the National Optical Astronomy Observatories, which are operated by the Association of Universities for Research in Astronomy, Inc., under cooperative agreement with the National Science Foundation. 


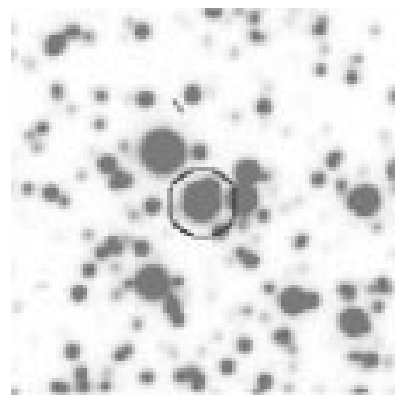

ve

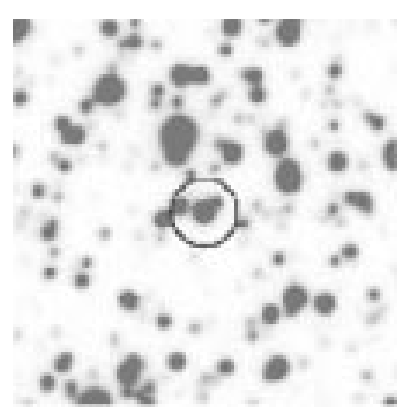

05

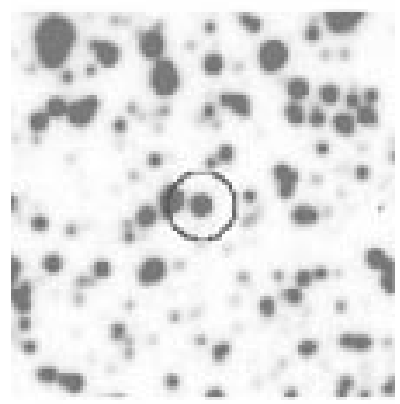

us

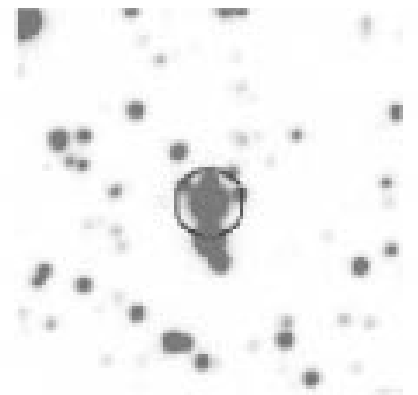

US

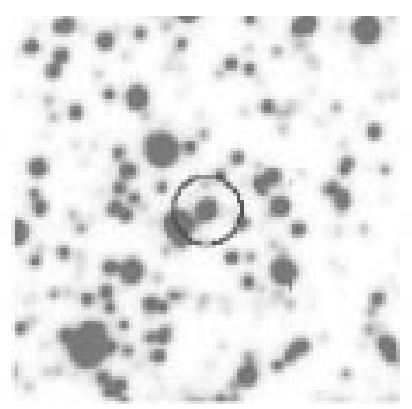

U6

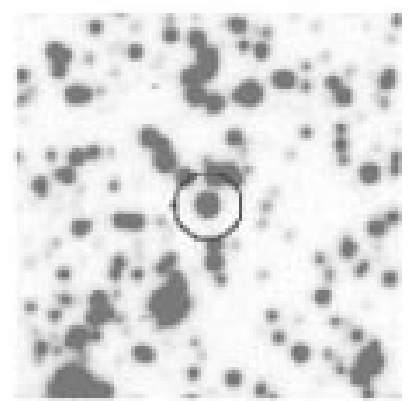

$\cup 9$

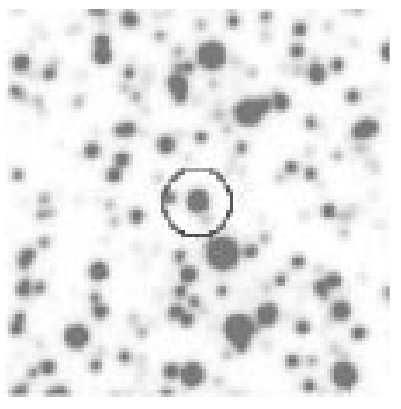

U4

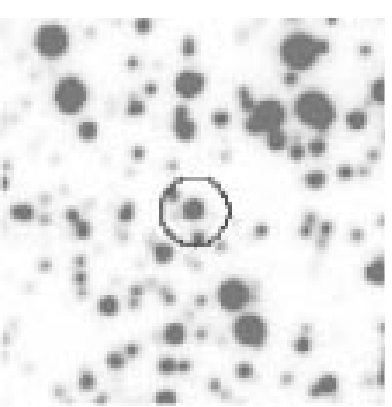

UP

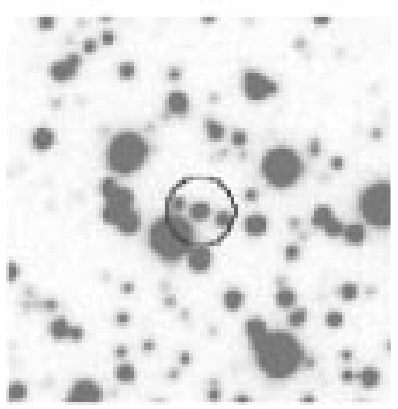

บ1ด

Fig. 1. Finding chart for NGC 288 variables. Each chart is $30 \operatorname{arcsec}$ on a side with north up and east to the left

described in some details in Kaluzny et al. (1995). The instrumental photometry was transformed to the $V$ system with the zero point defined by the data set from CTIO (Paper I).

\section{Variable stars}

To select potential variables we employed three methods which are described in detail in Kaluzny et al. (1996). The overall quality of photometry discussed here is similar to the quality of data discussed in Paper I (see Fig. 1 in that paper). The new data set is, however, by a factor of 6 more extended with respect to the total number of measurements available for a given star. An additional advantage is the fact that on several nights the cluster was monitored continuously for 4-6 hours. In contrast, during the CTIO run the longest session on NGC 288 lasted only about 1.5 hours.
Nine certain periodic variables were identified. This sample includes 6 already known objects and 3 newly discovered variables. A finding chart allowing unambiguous identification of all variables is shown in Fig. 1.

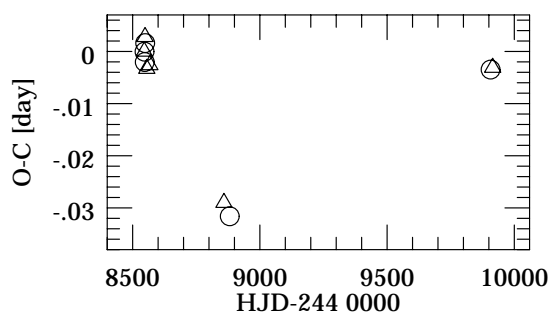

Fig. 2. The $\mathrm{O}-\mathrm{C}$ diagram for RR Lyr star V3. Circles and triangles correspond to moments of minimum and maximum light, respectively 

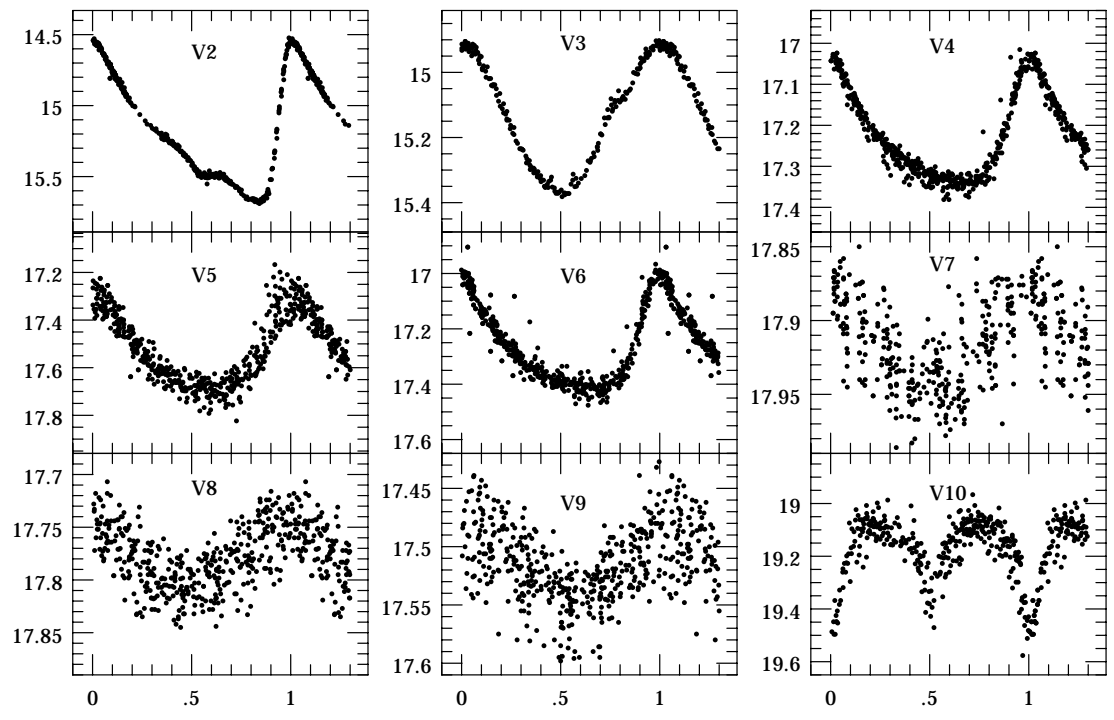

Fig. 3. Phased $V$ light curves for NGC 288 variables V2 - 10

Table 1 lists some basic characteristics of the light curves of variables $\mathrm{V} 2-10$. The periods were determined using ANOVA statistic (Schwarzenberg-Czerny 1996). The light curves used for period determination were expanded by including data reported in Paper I. The time base of these combined light curves equals 5 years. The periods of all variables are firmly established. The period of V3 has been substantially revised in comparison with the value given in Paper I. Moreover, our data indicate the presence of some peculiarities in the light curve of V3. We found it difficult to phase all observations of V3 with the single ephemeris. Examination of the periodogram obtained with ANOVA algorithm (Schwarzenberg-Czerny 1996) reveals the presence of two peaks of comparable height near the frequency $\nu=2.325$. Periodograms obtained for other variables show single peaks at frequencies corresponding to periods listed in Table 1 . It is known that some RR Lyr stars show long-term amplitude modulation. This behavior is known as Blazhko effect (Blazhko 1907). In fact, the amplitude of V3 seems to be very stable, so it is not a Blazhko variable. Our data indicate instead the presence of some phase shifts in the light curve of V3. This is illustrated in Fig. 2 where we show the $\mathrm{O}-\mathrm{C}$ diagram for the moments of maxima and minima of light. The $\mathrm{O}-\mathrm{C}$ deviations were calculated using ephemerides:

$$
\begin{gathered}
T_{\mathrm{Min}}=2448546.7374+E \times 0.430173 \\
T_{\mathrm{Max}}=2448545.6641+E \times 0.430173 .
\end{gathered}
$$

The presented $\mathrm{O}-\mathrm{C}$ diagram can be interpreted as an evidence for increasing period of V3. However, more data are needed to clear up this problem.

The colors listed in Table 1 are based on a single exposure in the $B$-band and two bracketing exposures in the $V$-band. The mean $V$ magnitudes were calculated by numerically integrating the phased light curves after converting them to an intensity scale. Table 1 gives also equatorial coordinates for variables V2 - 9. A transformation from rectangular to equatorial system was derived based on positions of 50 stars from the list provided kindly by Dr. Guo (Guo et al. 1993). These stars were identified in the outermost parts of the field covered by TEK3 camera. No transformation star could be located in the central part of the cluster. Therefore there is a possibility that the coordinates derived for variables from the central part of NGC 288 suffer from some systematic errors.

The phased light curves of stars $\mathrm{V} 2-10$ are shown in Fig. 3. For V3 we plotted only measurements obtained between October 16, 1991 and November 7, 1991. Variables V8 and V9 joint the group of 4 other SX Phe stars identified previously in NGC 288. Object V10 is most probably a contact binary of W UMa type. Figure 4 shows a CMD of NGC 288 with marked positions of variables $\mathrm{V} 2-10$. The presented data are based on photometry obtained with the Swope telescope and TEK2 camera. All SX Phe stars occupy positions among blue stragglers. The contact binary V10 is located slightly above the main sequence. We note that only very few binary stars located below turnoffs of their host globular clusters are known for a moment (Mateo 1996). In fact, a prevailing fraction of binaries identified so far in globular clusters are blue stragglers. In contrast, a large fraction of eclipsing binaries known in old open clusters is located on the red side of main sequence (e.g. NGC 188, NGC 2243 and M 67). This apparent difference between globular and open clusters results likely from a selection effect favoring a discovery of only relatively brighter systems in globular clusters. 
Table 1. Light-curve parameters for NGC 288 variables $\mathrm{V} 2-10 . \Delta V$ is the range of observed variations in the $V$ band. The period is given in days. The $B-V$ color was measured at random phases

\begin{tabular}{rrlllllll}
\hline Name & Type & $\begin{array}{l}\text { Period } \\
\text { day }\end{array}$ & $\langle V\rangle$ & $V_{\max }$ & $\Delta V$ & $B-V$ & $\begin{array}{r}\mathrm{RA}_{2000} \\
\text { h:m:sec }\end{array}$ & $\begin{array}{r}\text { Dec }_{2000} \\
\text { deg: }^{\prime}:^{\prime \prime}\end{array}$ \\
\hline V2 & RRab & 0.67775 & 15.18 & 14.55 & 1.12 & 0.38 & $0: 52: 46.64$ & $-26: 34: 07.4$ \\
V3 & RRc & 0.4302 & 15.14 & 14.92 & 0.45 & 0.28 & $0: 52: 40.21$ & $-26: 32: 28.8$ \\
V4 & SX & 0.07907 & 17.24 & 17.04 & 0.30 & 0.29 & $0: 52: 42.80$ & $-26: 34: 45.4$ \\
V5 & SX & 0.05107 & 17.54 & 17.33 & $0.46:$ & 0.16 & $0: 52: 44.98$ & $-26: 33: 52.1$ \\
V6 & SX & 0.06722 & 17.28 & 17.01 & 0.41 & 0.30 & $0: 52: 42.40$ & $-26: 34: 54.6$ \\
V7 & SX & 0.03996 & 17.92 & 17.88 & 0.06 & 0.28 & $0: 52: 41.39$ & $-26: 33: 59.7$ \\
V8 & SX & 0.04653 & 17.78 & 17.74 & 0.06 & 0.28 & $0: 52: 44.27$ & $-26: 33: 59.6$ \\
V9 & SX & 0.03937 & 17.52 & 17.49 & 0.05 & 0.29 & $0: 52: 42.88$ & $-26: 34: 09.6$ \\
V10 & EW & 0.43875 & - & 19.09 & 0.38 & 0.61 & $0: 52: 47.86$ & $-26: 33: 01.9$ \\
\hline
\end{tabular}

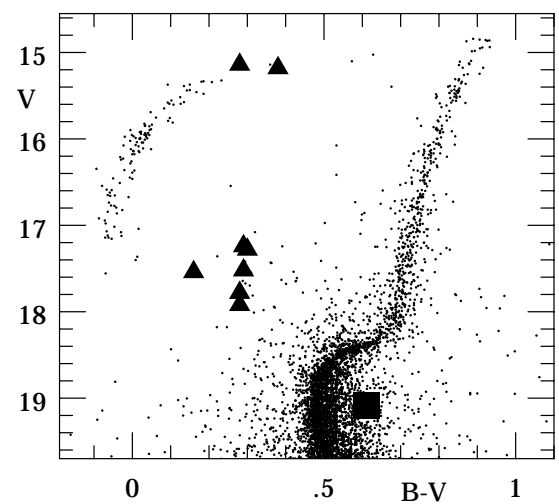

Fig. 4. The color-magnitude diagram for NGC 288 with the positions of the variables $\mathrm{V} 2-10$. The contact binary V10 is marked with a filled square

\subsection{Cluster membership of the variables}

It was already shown in Paper I that SX Phe stars V4 - 7 are likely members of the cluster. That conclusion was based on a good agreement between absolute magnitudes of these stars derived under the assumed cluster membership and absolute magnitudes calculated from the $p e$ riod versus luminosity relation as calibrated by McNamara (1995). The analogical procedure applied to variables V8 and V9 leads to the conclusion that they, too, are likely members of NGC 288.

We have applied the absolute brightness calibration of W UMa type binaries established by Rucinski (1995) to estimate $M_{V}$ for the newly discovered contact binary V10. Rucinski's calibration gives $M_{V}$ as a function of period, unreddened color $(B-V)_{0}$ and metallicity:

$$
\begin{aligned}
M_{V}^{\mathrm{cal}}=-2.38 \log (P) & +4.26(B-V)_{0} \\
& +0.28-0.3[\mathrm{Fe} / \mathrm{H}] .
\end{aligned}
$$

We adopted $[\mathrm{Fe} / \mathrm{H}]=-1.4$ and $E(B-V)=0.04$ for NGC 288 (Zinn 1985). We obtained from Rucinski's calibration $M_{V}=4.06$ for V10. The formal error of this es- timate, resulting purely from uncertainties in Rucinski's calibration, is about $0.3 \mathrm{mag}$. On the other hand we may estimate an absolute magnitude of the variable under assumed cluster membership. We adopted an apparent distance modulus of the cluster $(m-M)_{V}=14.5 \pm 0.2$ (Pound et al. 1987) and obtained for V10 $M_{V}=4.17$. The fact that the above two estimates of $M_{V}$ are consistent with each other supports the hypothesis that V10 is a member of NGC 288. We note also that the cluster location on the sky near the South Galactic Pole implies a small contamination of its field by the foreground stars.

\section{Summary}

We surveyed the central part of the globular cluster NGC 288 for a total of 64 hours and identified 3 new variables. Of particular interest is the discovery of a contact binary which is located slightly above the upper main sequence of the cluster. Very few main sequence binaries are known for a moment in the whole sample of galactic globular clusters. The remaining 2 of newly discovered variables are pulsating blue stragglers of SX Phe type. There are 6 known stars of this type in NGC 288. It is interesting to note that for 3 of them light amplitudes in $V$ are smaller than $0.10 \mathrm{mag}$. This is a bit unexpected because two recent lists of SX Phe stars (McNamara 1995; Nemec et al. 1995) contain only one object with $A_{V}<0.10$ mag. At the same time, the recent survey of $\omega$ Cen leads to identification of 43 SX Phe variables of which 26 show amplitudes $A_{V} \leq 0.10 \mathrm{mag}$ (Kaluzny et al. 1996, 1997). Based on a comparison of a sample of SX Phe stars discovered in $\omega$ Cen and in NGC 288 with a sample of field and cluster objects (McNamara 1995) we arrive at the conclusion that the later sample is significantly incomplete as regards the variables with low amplitudes. The Pop I counterparts of SX Phe stars are $\delta$ Sct variables. Variables with amplitudes as small as a few hundreds of magnitude prevail in the sample of bright $\delta$ Sct stars (Halprin \& Moon 1983). We predict that the currently conducted surveys of nearby 
clusters will result in the discovery of many SX Phe stars with very small amplitudes. Our data indicate fast changes of pulsation period for the RR Lyr star V3.

Acknowledgements. This project was supported by the Polish KBN grant 2P03D00808 to JK and by NSF grant AST9528096 to Bohdan Paczyński. We thank Dr. Xinjian Guo for providing us with equatorial coordinates of stars from the NGC 288 field.

\section{Appendix A}

Tables containing the light curves of all variables discussed in this paper are published by A\&A at the Centre de Données de Strasbourg, where they are available in electronic form.

\section{References}

Blazhko S., 1907, Astron. Nachr. 175, 325

Bolte M., 1992, ApJS 82, 145

Guo. X., Girard T.M., van Altena W.F., 1993, AJ 105, 2182

Halprin L., Moon T.T., 1983, Astroph. Sp. Sci. 91, 43
Kaluzny J., 1997, A\&AS (in print)

Kaluzny J., 1996, A\&AS 120, 83 (Paper I)

Kaluzny J., Kubiak M., Szymański M., et al., 1995, A\&AS 112, 407

Kaluzny J., Kubiak M., Szymański M., et al., 1996, A\&AS 120, 139

Kaluzny J., Kubiak M., Szymański M., et al., 1997, A\&AS 122, 471

Kaluzny J., Thompson I., Krzeminski W., 1997 (submitted to AJ)

Mateo M.M., 1996, in "The Origins, Evolution and Destinies of Binary Stars in Clusters", Milone E.F. \& Mermilliod J.-C. (ed.), ASP Conf. Ser. 90, 21

McNamara D.H., 1995, AJ 109, 1751

Nemec J.M., Linnell Nemec A.F., Lutz T.E., 1994, AJ 108, 222

Paczyński B., 1996, in The Extragalactic Distance Scale, STScI May Symposium

Pound M.W., Janes K.A, Heasley J.N., 1987, AJ 94, 1185

Rucinski S.M., 1995, PASP 107, 648

Schechter P., Mateo M., Saha A., 1993, PASP 105, 1342

Schwarzenberg-Czerny A., 1996, ApJ 460, L107

Stetson P.B., 1987, PASP 99, 191

Zinn R., 1985, ApJ 293, 424 